\title{
An Experienced Chinese Teacher's Strategies In Teaching Mathematics: Translation of Quadratic Functions
}

\author{
Xingfeng Huang, Jinglei Yang, Bingxing Tang, Lingmei Gong, Zhong Tian \\ Department of Mathematics, Changshu Institute of Technology, Jiangsu, China. \\ $<$ xfhuang0729@gmail.com>
}

\begin{abstract}
The study selected the topic of translation of quadratic functions. In order to explore some effective instructional strategies to help students understand this topic, an experienced teacher was chosen for a case study. Based on lesson observation and semi-structure interviews, this study found that the teacher employed various strategies to facilitate students understanding of translations of quadratic functions.
\end{abstract}

Keywords experienced Chinese teacher; strategies; translation of quadratic function

\section{Introduction}

With the background of Chinese curriculum innovation, how do mathematics teachers apply teaching strategies to classroom practice? This issue aroused researchers' interest. A lot of mathematics public lessons (in Chinese公开课) attracted their research (Huang, 2009; Huang \& Fan, 2009; Li \& Li, 2009, Huang \& Li, 2009). The purposes of these studies were not only to promote policy-makers to understand the implementation of curriculum innovation, but also to encourage teachers to have opportunities to learn while reflecting on their own teaching (Yu, 2002; Wong, 2009). However, there has been criticism of the model lessons. Some people argued that public lessons looked like certain shows which are pretty but not practical and that these lessons did not represent the real matter (Qian, 2007). With this in mind, it became important to pay special attention to teachers' strategies in their routine classroom practice. This study focuses on a junior school teacher with 10 years of teaching experience, and explores his teaching strategies in his routine classroom.

\section{Focus On A Challenging Topic}

As the evaluation of the teacher, as well as the evaluation of classroom teaching strategies, it is often rewarding if he completes a challenging task. It is a good opportunity for the teacher to exhibit his instructional wisdom, when he is given challenging tasks.

For secondary students, function is a difficult mathematics concept (Leinhardt, Zaslavsky, \& Stein, 1990; Sajka, 2003; Vinner \& Dreyfus, 1989). From grade 7 to 9, the curriculum introduces the specific function of three types: linear function, inverse function and quadratic function (Ministry of Education, 2001). The results show that students encounter a lot of cognitive obstacles in learning quadratic functions (Zaslavsky, 1997). Textbooks of grade 7 to 9 often include this topic, which is finally introduced in the algebraic field. In the chapter of quadratic function, translation of $y=\mathrm{ax}^{2}$ graph is an important 
component. The translation of quadratic functions includes vertical, horizontal and compound ones. In order to understand translation of quadratic function, first we must understand the concept of quadratic function. Because any translation of quadratic function is, by nature, an operation, students should think of quadratic function as an operational object. The order of instruction also brings obstacles to students' learning because they are usually taught vertical translation ahead of horizontal translation (Zazics, Liljedahl, \& Gadowsky, 2003). Vertical translation of quadratic functions coincides with students' intuition. For example, students are asked to translate $y=x^{2}$ to $y=x^{2}+1$. Comparing the two function expressions, if they add a positive one to $x^{2}$, the graph of $y=x^{2}$ will be moved up one unit in the positive direction along axis $y$. However, horizontal translation is against intuition. If $y=x^{2}$ is supposed to be translated to $y=(x+1)^{2}, x$ in the former expression is also added into a positive one, then the graph moved left toward in the negative direction along the axis $\mathrm{x}$. Since it is so different from vertical translation, it would be quite difficult for students to understand the concept of horizontal translation. A study by Eisenberg and Dreyfus (1994) shows it would be hard for students to understand horizontal translation. Baker, Hemenway, and Trigueros (2000) used APOS theory to explain students' learning difficulty. The vertical action is operated directly on a quadratic function, while the actions in horizontal are different, in which two operations are included, first on the variable, and then on the function.

\section{Research Question}

Recently some studies began to investigate Chinese curriculum innovation influences on mathematics classroom teaching (Huang, 2009; Huang \& Fan, 2009; Li \& Li, 2009, Huang \& Li, 2009). The main concern of these studies was to observe some excellent mathematics lessons, which were usually isolated, in which teachers were not observed in a structured way. In order to overcome the deficiencies of these studies, this study was grounded in curriculum innovation and conducted a series of successive observations of routine lessons, in which the focus was on the experienced teacher's strategies. In order to promote an understanding of the teacher's strategies, we chose a challenging topic of translation of quadratic functions. The research question was: What strategies does the experienced teacher use to help students understand the concept of translation of quadratic functions?

\section{Methodology}

\section{Participant}

Mr S graduated from the Department of Mathematics of a Normal university in 1999. He has 10 years teaching experience in Qin Chuan Junior School. He studied part-time for his Education Master Degree, and now he is preparing his dissertation. Qin Chuan Junior School covers about 66 acres, located in the west of Yucheng, which is a small city in the southern 
region of Jiangsu. This school has about 2,500 students divided into three grades from seven to nine. Each grade has 16 classes, and each class has 45 to 50 students mostly from local families.

We invited Mr S to participate in this study, because on the one hand he is an experienced teacher, and on the other hand, he and the researchers have a good relationship so as he can help us to complete the study successfully.

\section{Data collection}

From October to November 2009, Mr S's class of grade 9 was studied. The content of translation of quadratic functions was divided into three lessons, each of which has about 40minutes. In each mathematics classroom, a researcher videoed, and another wrote field notes. After each lesson, the lesson plan was copied, and the teacher was interviewed by the researcher using a semi-structured process where the researcher wrote notes. At the same time, the researcher who had videoed the lesson also recorded the whole process of the interview. Videos of the lessons and interviews were translated into scripts by four assistants.

In the next section a description is presented of how the experienced teacher used effective strategies to help students understand vertical translation of quadratic functions. His strategies have been identified to be similar with the other two lessons, the result of which is set aside for an appropriate occasion.

\section{Results}

\section{Review and foreshadowing (in Chinese铺垫)}

Before the lesson, Mr S copied a mathematics task on the blackboard. The task was: If the quadratic function $y=a x^{2}$ and the line $y=x-1$ have only one common point, then how many points of intersection do the function $y=4 a x 2$ and the line $y=x+3$ have?

$\mathrm{Mr} \mathrm{S}$ pointed out that the quadratic function could be denoted by an algebraic expression, but also could be represented by a graph. Therefore students could access solutions using two perspectives. He said: "We have learned features of function graph for some days, so I hope that you can use the graph features to solve this problem.” Next, the problem solving process was completed under the control of him. He asked his students to draw the two possible graphs of the quadratic function $y=a x 2$. At the same time he drew the two graphs of concave up and down on the blackboard.

The following are the teacher-student interactions. On the blackboard Mr S drew the graph, in which $\mathrm{y}=\mathrm{ax}^{2}$ and $\mathrm{y}=\mathrm{x}$-1have only one common point. Then Mr S asked students to stretch the graph of $y=a x^{2}$, and translate the graph of $y=x-1$ in the same system, so that they constructed the graph of $y=4 a x^{2}$ and $y=x+3$. Finally, the teacher guided his students to solve this problem by setting up an equation system.

In this teaching episode, based on the task, the class reviewed the relation between the opening direction and size of a parabola and its coefficient 'a' contained in the expression. On 
the other hand, translation of the linear function was employed to foreshadow the translation of the quadratic function.

Actually the teacher attempted to highlight the importance of the graph solution, although he guided his students to solve this problem by algebraic solution later. In the lesson, he gave a cue to his students: "We have learned the graph of a function, so I hope you can use graph features to solve this problem first of all." In the interviews, Mr S emphasized repeatedly that students lacked the idea of combining figures with graph (in Chinese数形结合). It is hard for students to associate the features of a function graph with its algebraic expression, and they felt it was difficult to learn quadratic functions. He also made it clear that he would always stress the idea of combining figures with graphs in teaching quadratic functions. He is convinced that if students generated this idea, they would be assured of understanding the content of quadratic functions, and be able to solve the problems related to it.

\section{Transition}

Mr S made a transit to the theme of this lesson, vertical translation of quadratic functions. He asked: "What is the relationship between the quadratic functions $y=a x^{2}$ and

$y=a x^{2}+b x+c$ ?" After a moment in silence, he told the students: "Special versus General". Then, Mr S asked his students to classify $y=a x^{2}+b x+c$ according to its coefficients whether it is zero or not. Then, he raised a question: "What is the relationship between the graph of $y=a x^{2}$ and $y=a x^{2}+b x+c$ ?"

In fact, it was found that the classification of the quadratic function was too difficult for the students. Although he gave his students a lot of cues, and gave them time to explore it, they did not finally succeed. The students' performance in the lesson surprised the teacher. After the lesson he said:

\section{I have spent too much time dealing with classification, which should not be stressed in this lesson. My intention was to enable students to realize the algebraic relation between $\mathrm{y}=\mathrm{ax}^{2}$ and $\mathrm{y}=\mathrm{ax}^{2}+\mathrm{c}$, and pilot them to consider the graph relation between them.}

His comment implies that he dealt with the classification of quadratic functions because of two points. Firstly, considering the pedagogy, he wanted to strengthen the coherence of the classroom instruction. Secondly, he emphasized the connection among $\mathrm{y}=\mathrm{ax} \mathrm{x}^{2}+\mathrm{bx}+\mathrm{c}, \mathrm{y}=\mathrm{ax} \mathrm{x}^{2}$ and $\mathrm{y}=\mathrm{ax} \mathrm{x}^{2}+\mathrm{c}$, in order to encourage his students to understand the concept of quadratic functions.

\section{Core concept}

Conjecture. The instruction focuses on the core concept of the lesson. Mr S posed the problem which should be solved in the lesson. He said: "We have learned the function of $y=a x^{2}$. Today we will explore the features of the function $y=a x^{2}+c$. These look quite alike. As to how obtain $y=a x^{2}+c$, just add $c$ to $y=a x^{2}$. What changes on the graph?" His students 
answered in unison: "Translate it upward or downward". It looked like a very obvious matter for them. Mr S invited a student to make the explanation. He asked: "Yun, can you explain?"

Yun: Just like the translation on linear function.

Mr S: Can you give us an example?

Yun: $y=2 x, y=2 x+1$

Yun made an analogy translation of linear function to translation of a quadratic one. Perhaps, the foreshadowing at the beginning of the lesson had an effect on their thinking. So the student could easily connect two type of translation with. Mr S said would like to hear other different answers.

Feng: Take a point on $y=a x^{2}$. Its abscissa is $\mathrm{x}$, while its ordinate is $a x^{2}$.

Mr S: Let $\left(\mathrm{x}_{1}, \mathrm{y}_{1}\right)$.

Feng: Substitute $\mathrm{x}_{1}$ in $\mathrm{y}=\mathrm{ax}^{2}+\mathrm{c}$, then get $\mathrm{y}_{1}+\mathrm{c}$.

Mr S: What is the position relation between the points of $\left(\mathrm{x}_{1}, \mathrm{y}_{1}\right)$ and $\left(\mathrm{x}_{1}, \mathrm{y}_{1}+\mathrm{c}\right)$ ?

Feng: Translate upward or downward.

In fact, the teacher emphasized the explanation of the graphical features on the algebraic perspective. In the previous lesson, he had always taken the trouble to lead his students to use point-coordinates to interpret the symmetry of $y=a x^{2}$. In the interview, he said:

\begin{abstract}
Algebraic explanation is the most powerful. When we see a function expression, we should think of its graphical features; when looking at function graph, we should explain it on the algebraic perspective.
\end{abstract}

It also shows that $\mathrm{Mr} \mathrm{S}$ always highlighted the idea of combining figures with graphs.

In this episode, he first attempted to give students an overall impression on the translation of quadratic function. He expected that students would preliminarily perceive the translation of a parabola before accessing details. This strategy which is consistent with the view of Gestalt could facilitate students to connect existing cognitive conceptions (translation of linear function) with the overall impression so as to establish the structure which can assimilate specific knowledge.

Depicting points to draw a parabola. Mr S requested his students to complete the mathematical task: Depicting points to draw parabolas of $y=1 / 2 x^{2}, y=\frac{1}{2} x^{2}+1$, and $y=\frac{1}{2} x^{2}-1$ at the same coordinate system, then discussing their features.

In the first step, the teacher listed a table on the blackboard, took $x=-3,-2,-1,0,1,2$, 3symmetrically, and found the corresponding value of $y=\frac{1}{2} x^{2}$ in order. He said: "Do you think the value of $y=\frac{1}{2} x^{2}+1$ must be calculated in this way? ... Yes, don't calculate any longer, as long as the latter's corresponding value of the $y=\frac{1}{2} x^{2}$ plus one." When they were 
to find the corresponding value of function $y=\frac{1}{2} x^{2}-1$, he also asked the students to focus their attention on the feature: as long as the last function's value is same from the function $y=\frac{1}{2} x^{2}$ but add negative one.

In the tabulation process, Mr S asked his students repeatedly to pay attention to the numerical relation among the three functions' values, in which the same number was taken from. In this way, he intended to help his students to understand the relation among the function graphs.

The teacher required each student to depict the graphs of $y=\frac{1}{2} x^{2}, y=\frac{1}{2} x^{2}+1$, and $y=\frac{1}{2} x^{2}-1$ on the cross-section paper. A few students drew exotic graphs: (1) The three parabolas' vertices coincided at the origin; (2) Parabolas intersected; (3) Parabolas extended in different directions, but did not intersect. Mr S saw several students drawing intersected parabolas, then required all students to watch the parabolas which he had drawn on the blackboard. He explained: "It is impossible for these parabolas to intersect, because each point on the graph of $y=\frac{1}{2} x^{2}$ is moved upward one unit, so as to get the graph of $y=\frac{1}{2} x^{2}+1$. Can the original graph intersect with the new one? No, they cannot.”

After the lesson, we told $\mathrm{Mr} \mathrm{S}$ the other errors in drawing the parabolas. He felt surprised that these students drew the three parabolas with the same vertex. He said:

\footnotetext{
If so, students still have no idea of translation? I already elicited students to conjecture. The purpose is to promote them to generate a overall impression on the translation. And I stressed repeatedly the change of the values in the table. Perhaps their impression on the graph of $y=a x^{2}$ is too deep -vertex must be at the origin.
}

Mr S had confidence that what he had done could enhance students' understanding of translation. He just contributed a few students' errors to their prior experience. So we can understand why the teacher employed similar strategies in the other two lessons on translation.

Because the teacher did not consider these errors in drawing before the lesson, his interpretation was confusing. In fact, a graph may intersect with the moved one. For example, a circle may intersect with the moved one. It was hard for him to make effective strategies to deal with unexpected accidents in classroom.

Depicting points in order to draw a parabola can be seen as the first stage of learning as Bruner (1967), namely the enactive mode. With concrete operations, students can take a quadratic function as an object, and construct the concept of translation based on their understanding. 
Sketch. The teacher required the students to sketch graphs of $y=-\frac{1}{2} x^{2}, y=-\frac{1}{2} x^{2}+1$, and $y=-\frac{1}{2} x^{2}-1$. As far as the teaching arrangement was involved, he explained:

At first, students can familiarize with the knowledge that they have just learned. At second, they have learned the parabolas of up-opening, and now they do an exercise on downopening. Furthermore, in solving problems we usually sketch rather than depict points.

The teacher's intention was obvious. On the one hand, he tried to make students understand the concept of translation with variation. On the other hand, he trained students' sketch skills, which could be used to solve problems. The teacher has a further understanding of the sketch value. He believed that conceiving a graph in the mind is more important than depicting points to draw it. Here, the former means the graph of a quadratic function operated in the mind, which is the second stage of Bruner's iconic mode, semi-concrete operation. The stage of iconic operation is important for students to understand the abstract concept of translation, the symbolic mode, which is the third learning stage defined by Brunner.

\section{Summary}

In classroom interactions, students observed similarities and differences between the graphs of $y=a x^{2}$ and $y=a x^{2}+c$, and then generalized the relation between them, so that they completed the third learning stage of symbolic mode.

In the instructional process of the core concept, the teacher firstly gave his students an overall impression of the translation process, and then accessed details. Students experienced the specific--semi-specific and semi-abstract-- abstract stage, in which they depicted points to draw a graph, or sketch, and generalized vertical translation of quadratic functions.

After that, there was no time to do other exercises in the class. However, the teacher's plan had not been completed. His intention before the lesson was to consolidate students' concept of the translation with some mathematics exercises.

\section{Conclusion}

In the lesson, the teacher emphasized the idea of combining figures with graphs, and highlighted the strategy of connecting algebraic representation with visualization. To be specific: (1) A mathematical task was selected to review what the students have learned, and prepare for learning new conception; (2) Foreshadowing and transit ion added to the lesson fluency, and different mathematics structures were connected; (3) the teacher gave students an overall impression of the core concept first, then accessed details; (4) The students learned the conception in a specific--semi-specific and semi-abstract-- abstract process; (5) The teacher intended to consolidate the concept with some mathematics exercises. 


\section{Acknowledgement}

This research was supported by Ministry of Education (China) under GOA107014.

\section{Reference}

Baker, B., Hemenway, C., \& Trigueros, M. (2000). On transformations of basic functions. In: H. Chick, K. Stacey, \& J. Vincent (Eds.), Proceedings of the $12^{\text {th }}$ ICMI Study Conference on the Future of the Teaching and Learning of Algebra (pp. 41-47). University of Melbourne.

Bruner, J. S. (1966). Toward a theory of instruction. Cambridge, MA: Harvard University Press.

Huang, X. F. (2009). Research on Mathematics Classroom in Shanghai. Nanning: Guangxi Education Publishing House.

Huang, X. F., \& Fan, L. H. (2009). Instructional practice in mathematics classroom driven by curriculum reform: a case study of model lesson from the Shanghai Two Round Curriculum Reform. Journal of Mathematics Education, 3, 25-30.

Huang, R., \& Li, Y. (2009).Pursuing excellence in mathematics classroom instruction through exemplary lesson development in China: a case study.ZDM-The International Journal on Mathematics Education, 41, 297-309.

Eisenberg, T., \& Dreyfus, T. (1994). On understanding how students learn to visualize function transformations. In: E. Dubinsky, A. Schoenfeld, \& J. Kaput (Eds.), Research in collegiate mathematics education 1, (pp. 45-68). Providence, RI: American Mathematical Society.

Leinhardt, G., Zaslavsky, O., \& Stein, M. K. (1990). Function, graphs, and graphing: Tasks, learning, and teaching. Review of educational Research, 60, 1-64.

Li, Y., \&Li, J. (2009). Mathematics classroom instruction excellence through the platform of teaching contests. ZDM-The International Journal on Mathematics Education, 41, 263277.

Ministry of Education. (2001). Mathematics Curriculum Standards in Compulsory Education. Beijing: Beijing Normal University publishing House.

Qian, W. W. (2007). Commentary of several contentious issues about open class.Shanghai Research On Education, 7, 34-37.

Sajka, M. (2003). A secondary school students' understanding of the concept of function: a case study. Educational Studies in Mathematic, 53, 229-254.

Vinner, S., \& Dreyfus, T. (1989). Images and definitions for the concept of function.Journal for Research in Mathematics Education, 20, 356-366.

Wong, N. (2009). Exemplary mathematics lessons: What lessons we can learn from them? ZDM-The International Journal on Mathematics Education, 41, 379-384.

Yu, P. (2002). Briefly on dual-function of publicly-given class. Shanghai Research On Education1, 31-33.

Zaslavsky, O. (1997). Conceptual obstacles in the learning of quadratic functions.Focus on Learning Problems in Mathematics, 19, 20-44.

Zazics, R., Liljedahl, P., \& Gadowsky, K. (2003). Conceptions of function translation: obstacles, intuitions, and rerouting. Journal of Mathematical Behavior, 22, 437-450. 